\title{
Metodologia de implementação de práticas preventivas ao uso de drogas na atenção primária latino-americana
}

\author{
Pedro Henrique Antunes da Costa, ${ }^{1}$ Daniela Cristina Belchior Mota, ${ }^{1}$ \\ Erica Cruvinel, ${ }^{1}$ Fernando Santana de Paiva ${ }^{1}$ e Telmo Mota Ronzani ${ }^{1}$
}

Como citar Costa PHA, Mota DCB, Cruvinel E, Paiva FS, Ronzani TM. Metodologia de implementação de práticas preventivas ao uso de drogas na atenção primária latino-americana. Rev Panam Salud Publica. 2013;33(5):325-31.

RESUMO Objetivo. Desenvolver uma metodologia para implementação de práticas de prevenção ao uso de álcool e outras drogas no âmbito da atenção primária à saúde (APS) que contribua com o debate sobre ações e políticas nos países latino-americanos.

Métodos. Trata-se de uma pesquisa-intervenção realizada em um município brasileiro de pequeno/médio porte. O processo foi avaliado através da observação participante, visando adequação às necessidades locais e ressaltando pontos de facilitação e dificuldade na implantação.

Resultados. Foi desenvolvido um modelo com seis etapas: contato inicial e planejamento, diagnóstico e mapeamento, sensibilização, capacitação, acompanhamento e devolutiva. Foram percebidos os seguintes pontos de dificuldade: insuficiência de recursos (humanos, financeiros, infraestrutura), falta de integralidade e intersetorialidade da rede assistencial, falta de participação dos médicos, formação calcada no saber médico, participação insuficiente da gestão de saúde, falta de mobilização e participação da sociedade civil, ausência de momentos onde a população fosse convidada a participar do planejamento e execução das politicas públicas. Pontos fortes foram: participação dos agentes comunitários e enfermeiros na aplicação, organização e planejamento das práticas, além da realização de práticas educacionais e preventivas nas escolas e comunidades pelas equipes de saúde. Isso indica que é possível implementar iniciativas de triagem, intervenção breve e encaminhamento para tratar (SBIRT) no contexto da APS latino-americana.

Conclusões. A metodologia desenvolvida neste estudo pode ser útil para países latinoamericanos desde que sejam consideradas as necessidades locais. Entretanto, os resultados serão observados apenas a médio e longo prazo, sem mudanças instantâneas.

Palavras-chave Atenção primária à saúde; pesquisa participativa baseada na comunidade; detecção do abuso de substâncias; América Latina.

Em todo o mundo, o uso de álcool e de outras drogas contribui para configurar um novo perfil no quadro dos problemas de saúde. Segundo a Organização Pan-

Universidade Federal de Juiz de Fora (UFJF), Departamento de Psicologia, Centro de Referência em Pesquisa, Intervenção e Avaliação em Álcool e Drogas (CREPEIA), Juiz de Fora (MG), Brasil. Correspondência: Telmo Mota Ronzani, tm.ronzani@gmail.com
Americana da Saúde (OPAS), em torno de 4,5 milhões de homens e 1,2 milhão de mulheres na América Latina e Caribe, em algum momento de suas vidas, sofreram transtornos causados pelo uso de drogas (1). De acordo com a literatura, os mecanismos mais efetivos na redução da vulnerabilidade da população a essas novas epidemias são aqueles que operam na prevenção das doenças e na promoção da saúde (2). Contudo, as experiências preventivas ao uso de álcool e outras drogas ainda são limitadas, apesar da importância de tais ações para mudar os indicadores de saúde geral da população.

Nessa perspectiva, diversos autores (3-5) apontam o enfoque denominado 
SBIRT - Screening, Brief Intervention, and Referral to Treatment (Triagem, Intervenção Breve e Encaminhamento para Tratar) - como uma importante ferramenta para a implementação e a organização de atividades preventivas ao uso de álcool e outras drogas, principalmente nos serviços de atenção primária à saúde (APS). Esse nível de atenção se configura como uma das principais estratégias de organização dos sistemas de saúde, principalmente na América Latina (6). É, portanto, uma instância essencial para a aplicação das práticas de prevenção ao uso de álcool e outras drogas. No Brasil, a APS é organizada através da Estratégia Saúde da Família (ESF), cujos principais atributos são: caráter substitutivo em relação à rede de atenção básica tradicional, atuação territorializada, desenvolvimento de atividades com foco na família e comunidade, busca da integração com instituições e organizações sociais e ser um espaço de construção de cidadania (7). Apesar disso, são escassos os estudos sobre a eficácia das práticas de prevenção ao uso de álcool e outras drogas na APS, com identificação de elementos que promovam resultados positivos na prevenção e redução do consumo de acordo com cada realidade (4).

Um dos componentes da SBIRT é a aplicação de instrumentos de triagem, como o teste para detecção de envolvimento com álcool, tabaco e outras substâncias (Alcohol, Smoking and Substance Involvement Screening Test, ASSIST), desenvolvido em 2002 pela Organização Mundial da Saúde (OMS) para identificar o padrão de uso e auxiliar o profissional de saúde a definir a estratégia de intervenção mais adequada (8). Após a aplicação do instrumento de triagem, realiza-se a intervenção breve, uma abordagem de educação em saúde relacionada à prevenção primária ou secundária para usuários de álcool e outras drogas, com foco na mudança de comportamento do paciente. Essa intervenção breve pode ser realizada por profissionais de diferentes formações (9). Contudo, no Brasil e em outros países latino-americanos, grande parte dos profissionais sente-se despreparada para lidar com a prevenção e a promoção de saúde, devido, entre outros fatores, à sua formação centrada na resolutividade da "doença" $(5,10)$. No que se refere à formação profissional, é importante o investimento na contínua formação desses atores sociais, a partir dos princípios da educação permanente, problematizando as demandas sociais emergentes e considerando as condições socioeconômicas e estruturais dos profissionais e usuários dos serviços de saúde $(11,12)$.

Nesse sentido, ressalta-se que os avanços científicos repercutem nas pessoas não exclusivamente pela atuação dos profissionais de saúde, mas também pelo impacto positivo que têm nos conhecimentos, valores e comportamentos das comunidades e sistemas sociais (13, 14). Para uma implementação efetiva das estratégias de prevenção, torna-se imprescindível a adoção de uma lógica de interação entre o sistema de saúde e a sociedade, a partir da compreensão das reais demandas e necessidades sociais.

Para tanto, as ações no campo da saúde devem ser compartilhadas com outros setores estratégicos, assim como devem garantir a participação efetiva dos diferentes grupos sociais na identificação, formulação e implementação das ações, ou seja, devolvendo o poder de decisão de sua saúde à própria comunidade $(13,15,16)$. A participação comunitária pode constituir eixos para a promoção de saúde e a prevenção do uso de álcool e outras drogas, pois as características ecológicas, culturais e socioeconômicas da comunidade na qual se pretende intervir passam a ser melhor visualizadas, configurando formas mais efetivas para que tais estratégias de prevenção atinjam a população $(16,17)$.

Cabe destacar ainda que, de modo geral, muitos projetos e ações são implantados sem que se avalie sua efetividade ou que se determinem fatores que possam facilitar ou dificultar sua implantação. Os aspectos científicos, tecnológicos e metodológicos imbricam-se com aspectos éticos e culturais de maneira indissolúvel, devendo ser considerados, desde o início, de maneira integral no ciclo completo da implementação das estratégias de prevenção ao uso de álcool e outras drogas (13).

Dessa forma, o objetivo do presente artigo é desenvolver uma metodologia para implementação de práticas de prevenção ao uso de álcool e outras drogas na APS. Para isso, foram determinados fatores que pudessem facilitar ou dificultar sua implantação, promovendo aproximação com a realidade local.

\section{MATERIAIS E MÉTODOS}

Esta pesquisa-intervenção de natureza qualitativa foi conduzida em um município brasileiro de pequeno/médio porte de março a dezembro de 2010 . O município possui 177 estabelecimentos cadastrados no Sistema Único de Saúde (SUS), sendo 147 da iniciativa privada e 30 de responsabilidade do poder público municipal (18). O município conta com 21 equipes de ESF na zona urbana e um Núcleo de Apoio à Saúde da Família (NASF) que atua em conjunto com os profissionais da ESF no matriciamento e organização do fluxo de pacientes. A média de profissionais por equipe de ESF é de 9,73 , sendo $76,5 \%$ da população coberta pela ESF (19). Todas essas equipes de ESF, além do NASF, foram abrangidas pelo estudo.

Quanto à rede de atenção aos usuários de álcool e outras drogas, o município dispõe de um Centro de Atenção Psicossocial para Álcool e Drogas (CAPSad). Entre os grupos de autoajuda, eram cadastrados na Secretaria Nacional de Políticas sobre Drogas (SENAD) quatro grupos de Alcoólicos Anônimos (AA) e dois grupos de Narcóticos Anônimos (NA) (20). Na rede particular foram encontradas uma clínica de repouso e duas comunidades terapêuticas. Além disso, existia no município o Conselho Municipal de Políticas para Álcool e Drogas, que, no entanto, encontrava-se desativado.

No município, assim como no restante do país, há uma sistematização do acesso aos serviços de álcool e outras drogas, com um protocolo de acolhimento, avaliação, referência e contra-referência dos pacientes que procuram os serviços de atenção primária à saúde. Todo paciente, para entrar na rede assistencial de saúde mental do município, deve ser avaliado inicialmente pelas equipes de ESF, exceto pacientes atendidos pelo serviço de urgências psiquiátricas.

\section{Participantes}

Participaram do projeto três gestores do sistema municipal de saúde, com base na importância dos seus cargos para a organização e a implementação do projeto (amostra intencional), e os profissionais das equipes de ESF e NASF que aceitaram participar de todas as etapas do projeto (amostra de conveniência). Além disso, foi enfatizada a participação de multiplicadores, que eram profissionais da própria rede e atores comunitários com uma inserção e/ou algum tipo de liderança/engajamento nas comunida- 
des, para auxiliar na execução do projeto junto às equipes e população.

\section{Avaliação do trabalho}

O processo foi avaliado visando a adequação às necessidades locais e examinando os fatores que pudessem facilitar ou dificultar a sua implantação em outros municípios e/ou países latinoamericanos. A partir do exposto, e de acordo com os achados na literatura, a metodologia de implementação de práticas preventivas foi avaliada através da observação participante. Os dados coletados foram registrados em diários de campo, gerando, posteriormente, relatórios sistemáticos (21). Além disso, reuniões semanais de planejamento foram efetuadas, sendo a avaliação do trabalho realizada continuamente.

\section{RESULTADOS}

Os resultados são apresentados em duas categorias: 1) metodologia de implementação das práticas preventivas ao uso de álcool e outras drogas e 2) pontos de dificuldade e pontos de facilitação observados no desenvolvimento dessa metodologia.

\section{Metodologia de implementação de práticas preventivas ao uso de álcool e outras drogas}

A metodologia de implementação foi desenvolvida a partir de seis etapas, sendo elas: contato inicial e planejamento, diagnóstico e mapeamento, sensibilização, capacitação, acompanhamento e devolutiva.

Na fase de contato inicial, a posição de colaboração entre todos os participantes foi corroborada, de modo que as ações propostas foram de responsabilidade mútua e realizadas em parceria. Tal pactuação ocorreu entre os meses de março e abril. Envolveu a elaboração, pelos pesquisadores e gestores de saúde, das estratégias de implementação do projeto, respeitando as características locais.

$\mathrm{Na}$ etapa de diagnóstico, iniciou-se um trabalho de inserção e familiarização ao campo e coleta de informações acerca das características locais. Tal processo teve duração de 2 meses e compreendeu a coleta de dados em sites do governo, Ministério da Saúde e Secretaria Nacional de Políticas sobre Drogas do Ministério da Justiça (Senad/MJ). Também foram coletados documentos sobre APS e ESF do município, com participação em reuniões da gestão de saúde com os gerentes de equipe de Saúde da Família e visitas às equipes de saúde e às comunidades.

Foram mapeados e analisados: os indicadores sociais e de saúde do município (dados epidemiológicos e de vulnerabilidade social), o cotidiano dos serviços de saúde (estrutura e dinâmica de funcionamento), a percepção e as demandas populacionais em relação ao serviço de saúde, a percepção dos profissionais em relação à população, os serviços e ações existentes para prevenção e tratamento do uso de álcool e outras drogas e o nível de engajamento e participação da sociedade civil no planejamento e implantação de políticas públicas. Tais dados foram analisados concomitantemente ao processo de coleta. Eles serviram como parâmetro para o contínuo desenvolvimento dos trabalhos.

Ainda nesta etapa, posteriormente à realização do mapeamento, foram identificados os multiplicadores. Além disso, foram promovidas parcerias com diversos setores identificados dentro da rede de apoio aos usuários.

A terceira etapa do projeto consistiu num trabalho de sensibilização dos vários atores sociais envolvidos na temática de álcool e outras drogas (gestores, profissionais da rede assistencial, líderes comunitários, etc.). Foi organizado um evento com duração de 1 dia que contou com participação desses atores, onde os pesquisadores do grupo e os gestores locais proferiram palestras sobre a importância da prevenção ao uso de álcool e outras drogas.

Contudo, o trabalho de sensibilização foi contínuo, com o objetivo, ao longo do tempo, de modificar crenças e atitudes moralizantes sobre o uso de álcool e outras drogas. Portanto, preconizou-se a disseminação e a troca de informações ao longo do projeto, remetendo, mais uma vez, à importância dos multiplicadores como ferramenta de alcance a uma maior parcela da população.

Na quarta etapa, os profissionais da ESF passaram por uma capacitação que incluiu noções teóricas sobre substâncias psicoativas (farmacologia, epidemiologia do uso, efeitos agudos e crônicos) e atividades práticas da SBIRT, como a aplicação do instrumento de triagem (ASSIST) e a técnica de IB. A capacitação ocorreu dentro da perspectiva da educa- ção permanente, com objetivos práticos e voltados para o processo de aprendizagem dinâmico e vivenciado no dia-a-dia dos serviços.

A quinta etapa do projeto foi o acompanhamento do processo de implementação das práticas de prevenção ao uso de álcool e outras drogas, promovendo uma articulação entre APS e comunidade local e mediando os interesses dos atores envolvidos em relação às ações sobre álcool e outras drogas. Para isso, durante 4 meses foram realizadas, simultaneamente: visitas quinzenais às equipes da ESF, entrega de materiais didáticos e educativos aos profissionais de saúde e reuniões semanais com os gestores da APS, com dois retornos parciais.

Através dos contatos com a gestão da APS, disponibilizou-se uma assessoria para a definição de uma agenda de ações sobre álcool e outras drogas, articulando os principais atores sociais no âmbito local.

Nas visitas às equipes de ESF, foi realizado o acompanhamento da aplicação das técnicas de prevenção pelos profissionais de saúde e uma discussão posterior dos casos encontrados, promovendo uma educação continuada dos conteúdos abordados na etapa de capacitação. Ofereceu-se suporte para o desenvolvimento das estratégias de prevenção. Também foram propostas estratégias voltadas para fatores organizacionais e processos de trabalho que poderiam impactar favoravelmente a realização das práticas de prevenção entre as equipes de profissionais de saúde.

Os dois retornos parciais consistiram em apresentações dos principais resultados referentes ao processo de implementação do projeto para a discussão coletiva dos fatores que facilitaram e/ ou dificultaram a implementação da proposta no município. O público alvo foi de profissionais da ESF, da rede de álcool e outras drogas, coordenação da APS e saúde mental. Essas apresentações tiveram o objetivo de planejar as próximas etapas do projeto, consolidando a sua implementação e o fortalecimento de parcerias locais estabelecidas.

Os materiais didáticos e educativos foram disponibilizados para os profissionais e usuários da ESF e continham dados sobre epidemiologia do uso de álcool e outras drogas e a realização da triagem e IB. O intuito dessa estratégia era de continuar com o processo de disseminação de informação e sensibiliza- 
ção e, também, servir como material de apoio para os profissionais na realização das práticas dadas na capacitação.

A sexta etapa foi executada a partir de um relatório final e de um último retorno. A finalidade foi sistematizar o projeto como um todo, apontando os pontos de dificuldade e facilitação à implementação das ações de prevenção ao uso de álcool e outras drogas. Ainda foram delineados os pontos que poderiam auxiliar no planejamento e continuidade das ações realizadas. No retorno final, foi arquitetada uma reunião com profissionais da ESF, da rede de álcool e outras drogas, coordenação da APS e saúde mental e demais interessados. $\mathrm{O}$ relatório final foi entregue à gestão e disponibilizado aos profissionais e demais interessados.

\section{Pontos de facilitação e dificuldade observados no desenvolvimento da metodologia}

A seguir serão apresentados os pontos de facilitação e dificuldade observados no desenvolvimento da metodologia, levando em consideração cada etapa de implementação.

Em relação ao contato inicial e o planejamento, estes foram facilitados por uma postura receptiva da gestão de saúde do município. Essa postura possibilitou a realização de um trabalho conjunto de planejamento e operacionalização das ações preventivas a serem realizadas pelos profissionais de saúde.

Na segunda etapa, de diagnóstico e mapeamento, constatou-se a existência de outros projetos sobre álcool e outras drogas - por exemplo, um grupo de trabalho composto por gestores de diversos setores e um fórum intersetorial permanente de saúde mental. Nesses projetos, as práticas realizadas no município eram apresentadas e discutidas entre usuários e profissionais da rede em encontros quinzenais. Foram realizadas parceiras com esses projetos preexistentes, o que contribuiu não apenas para aprofundar o conhecimento acerca das características da rede local, mas também para dar início ao processo de sensibilização. Contudo, a despeito dessas tentativas, foram observadas dificuldades de articulação da rede, com as noções de trabalho intersetorial baseadas apenas na junção de diferentes setores para a realização de ações com caráter pontual.
Ademais, na etapa de diagnóstico, foram observados outros pontos de dificuldade, entre eles: insuficiência de recursos na ESF, visão da população como mera receptora de políticas públicas, baixo número de contingente profissional nas equipes de saúde, infraestrutura inadequada dos postos de saúde e ausência de mobilização e participação da população no planejamento e realização de ações.

Na etapa de sensibilização, a participação da gestão de saúde e de outros setores ocorreu em momentos pontuais, como palestras e reuniões gerais com os profissionais de saúde e população. O grupo enfrentou dificuldades na mudança de crenças, atitudes e comportamentos dos profissionais, que eram muitas vezes pautados por preconceitos e estigmas sobre os usuários de álcool e outras drogas.

Na etapa de capacitação, 120 profissionais da ESF foram capacitados para definir e implementar as ações de prevenção ao uso de álcool e outras drogas. Foi notável a participação maciça de agentes comunitários de saúde, com 76 capacitados, e a participação escassa dos médicos, com somente 2 capacitados. Observouse, nas propostas de ações formuladas pelos profissionais da ESF, o foco na dependência, demonstrando uma formação ainda calcada no saber médico, curativo, que não priorizava a prevenção.

Ainda quanto ao processo de acompanhamento das equipes de ESF, foi destacada a participação de agentes comunitários de saúde na aplicação das práticas preventivas, e dos enfermeiros na organização da SBIRT. Foi percebida também uma ausência dos médicos nesta etapa. Os pontos facilitadores observados foram a realização da SBIRT pelos profissionais da ESF, aplicando o ASSIST e realizando a intervenção breve; e as práticas educacionais e preventivas realizadas em escolas por algumas equipes de ESF, como cursos, palestras, feiras e exposições. Essas práticas ocorreram a partir da aproximação das equipes de ESF com escolas de suas áreas de abrangência e de acordo com as necessidades e realidades locais. Ademais, algumas equipes planejaram e implementaram grupos nas comunidades para discussão de diversos aspectos sobre o uso de álcool e outras drogas, como as consequências do uso e da prevenção, entre outros.

Contudo, os profissionais envolvidos relataram ainda haver receio e falta de suporte da gestão municipal para a abordagem das questões ligadas ao tema. Foram também relatados problemas no encaminhamento de pacientes - com sobrecarga para o CAPSad, que não conseguia suprir a demanda de tratamento do município, e (mais uma vez) a falta de intersetorialidade, com os setores atuando, em sua maior parte, de forma isolada, sem articulação.

Por fim, na etapa devolutiva, observaram-se, como pontos facilitadores, a postura receptiva da gestão em relação aos resultados apresentados pelo projeto. $\mathrm{Na}$ reunião final, houve participação ativa dos agentes comunitários de saúde e dos enfermeiros na discussão dos pontos de facilitação e dificuldade observados a partir de cada etapa de implementação da metodologia. Ademais, uma profissional da ESF que teve atuação destacada foi selecionada para supervisionar a execução do projeto e para apoiar a implantação e a continuidade do modelo de prevenção proposto. A nomeação dessa profissional foi pactuada com a gestão de APS.

\section{DISCUSSÃO}

Ao se pensar numa metodologia de pesquisa-intervenção, algumas características inerentes às realidades e necessidades locais, além da própria metodologia de trabalho e do papel do pesquisador, devem ser analisadas, para que não haja simplesmente uma transposição de modelos fora do contexto. Mesmo que exista um planejamento inicial à entrada no campo, esse planejamento deve ser maleável e modificável a partir do mapeamento de demandas e dificuldades dos serviços e da população, da definição das problemáticas a serem consideradas e da possibilidade de resolução ou enfrentamento dessas problemáticas. Todo esse processo deve ser calcado na participação conjunta, relações dialógicas estabelecidas entre profissionais e comunidade, possibilitando a construção de novos saberes $(22,23)$.

Com relação à APS no Brasil e em grande parte da América Latina e Caribe, a literatura demonstra que, apesar de profissionais e gestores atribuírem grande importância às ações de prevenção e promoção de saúde, essas ainda ocorrem de forma paralela. $\mathrm{O}$ discurso preventivo, embora amplamente discutido na teoria, raramente é colocado em prática nos serviços de saúde (10). No 
Brasil, a população ainda procura as equipes de ESF com demandas majoritariamente de cunho curativo e remediativo - por exemplo, consultas médicas e odontológicas, requisição de medicamentos e realização de curativos e vacinas. A lógica que orienta o trabalho dos profissionais tende a ser o da legitimação de tais demandas sociais $(20,24)$. Cabe salientar que isso é fruto de um processo sócio-histórico com várias formulações e reformulações. Nesse processo, é recente a introdução dos conceitos de prevenção e promoção de saúde, tanto para a população quanto para os profissionais, em comparação aos pressupostos da lógica biomédica (20).

Aliados a isso estão os problemas referentes à formação dos profissionais de saúde e suas práticas. O modelo de APS exige uma mudança estrutural em ambas, que deve começar nos centros formadores. Contudo, ainda percebemse os profissionais de nível superior com sua formação e atuação voltadas para os processos de adoecimento (10). Ademais, a APS aparece como um meio importante de inserção no mercado de trabalho, especialmente para aqueles em início de carreira, estando a permanência desses, muitas vezes, condicionada pela falta de outras oportunidades de trabalho (24).

No caso dos agentes comunitários de saúde (ACS), muitos não tiveram passagem alguma pela área da saúde anteriormente, adquirindo na prática o seu real aprendizado, acarretando uma reprodução de modelos de pensamento e ação hegemônicos. Portanto, o trabalho de prevenção ao uso de álcool e outras drogas não pode mostrar-se desconexo dessas análises conjunturais, com o objetivo de uma maior conscientização da população, de profissionais e de gestores (24).

Outros fatores também são inerentes à APS no contexto latino-americano, como a insuficiência de recursos (humanos, financeiros, infraestrutura) e a falta de integralidade e intersetorialidade da rede assistencial $(6,25)$. Tais problemas foram observados no município estudado, gerando grande demanda de atendimento, limitando e, em alguns casos, impossibilitando as ações de prevenção. Entretanto, algumas ações pensadas e concretizadas pelas equipes de saúde, além da aplicação das práticas de SBIRT, indicam tentativas mais amplas de se pensar na temática de álcool e outras drogas. Isso adveio de um maior aprofundamento e conscientização de alguns profissionais sobre o conceito de saúde e suas práticas, mostrando também como é possível realizar ações coletivas, mesmo em situações que não sejam as ideais.

Destaca-se para isso a necessidade de parcerias com atores/entidades locais, nas quais esses atores assumam a responsabilidade de aumentar a capilaridade da divulgação da prevenção ao uso de álcool e outras drogas e de oferecer suporte à implementação das práticas de acordo com as potencialidades e peculiaridades de cada localidade, promovendo o engajamento da comunidade. A posição de destaque exercida pelos profissionais da ESF perante a comunidade - principalmente os enfermeiros e os ACS - confere a esses profissionais um papel de elo entre o serviço e a população, sendo que, além disso, participam de forma ativa do contexto das equipes. Ao se objetivar a continuidade das ações propostas na rotina de trabalho, é preciso considerar a comunidade como o componente principal, com os profissionais gerenciando e organizando as ações em suas respectivas equipes e áreas de abrangência (16).

Entretanto, deve-se aproximar a equipe e a rede assistencial para um trabalho interdisciplinar/intersetorial. Sabe-se que a questão do uso de álcool e outras drogas remete a diversas causas e dimensões, não sendo resolvida apenas pelo esforço setorial isolado da saúde. Articulações intersetoriais e a atuação multiprofissional são imprescindíveis para incidir sobre os determinantes sociais do processo de uso de álcool e outras drogas e promover a saúde (26).

Com relação às práticas de SBIRT, estas devem acompanhar a rotina de trabalho dos profissionais, ainda que em momentos de maior sobrecarga de trabalho tais práticas sejam realizadas com menor frequência. Daí a importância do planejamento e do estabelecimento de metas concretas na APS. Essas ações, aliadas a maior e melhor investimento, melhorias das condições de trabalho, suporte às equipes de saúde, são condições para a sustentação e melhoria dos serviços ofertados.

Pelo fato de serem práticas relativamente novas e que requerem mudança de paradigmas, grande parte dos profissionais de saúde tem receio de abordálas dentro de suas comunidades (12). Frente a isso, é necessária uma postura compreensiva, sugerindo soluções como a aplicação do ASSIST e a realização da IB em pessoas mais próximas, a fim de uma maior ambientação do profissional às práticas.

Também se mostra importante para manutenção das práticas preventivas a realização de um planejamento das equipes de saúde para a implementação da SBIRT em seus serviços, abordando a maneira como serão realizadas, estabelecendo metas e funções de cada profissional e pontuando possíveis entraves e pontos facilitadores a serem enfrentados de acordo suas realidades (12).

Sobre o papel da gestão, percebe-se na literatura uma dificuldade dos gestores em ponderar adequadamente a importância de ações preventivas ao uso de álcool e outras drogas (27). No município, observou-se a gestão de saúde receptiva à realização dessas práticas preventivas dentro da rotina dos serviços de saúde. Entretanto, não foi possível perceber um engajamento com as práticas propostas, como se o projeto não estivesse sob a responsabilidade do município também, numa perspectiva articuladora da atuação dos profissionais de saúde. Isso se torna um fator que dificulta a incorporação e manutenção das práticas na rotina assistencial do sistema de saúde local (27).

Os processos de construção das políticas públicas de saúde do município foram observados como sendo, em sua maioria, de cima pra baixo, onde profissionais e população são vistos como agentes passivos, não sendo convidados para o processo de construção dessas políticas. Isso nos remete à proposta da participação comunitária num contexto latino-americano que, segundo BriceñoLeon (28), trata-se de uma maneira de fortalecer a democracia. A sociedade civil há de assumir os seus deveres e implicações na construção do setor saúde, mas também não se pode acusá-la somente por uma ausência de participação quando o Estado não se movimenta para criar um espaço de debate, convidando a sociedade a participar como agente fomentador de reflexões e mudanças (28).

\section{Conclusões}

Algumas limitações foram percebidas durante o projeto, relacionadas, principalmente, às concepções da população e profissionais sobre o uso de álcool e outras drogas e às questões referentes à estruturação e dinâmica de funciona- 
mento da rede de APS. Apesar disso, considera-se o método apresentado e avaliado no presente artigo como um modelo útil para ser adotado, com suas devidas adaptações aos países latinoamericanos. Ademais, demonstra que, apesar do incentivo cada vez mais frequente dos programas de capacitação, estes de tornam limitados para a mudança de fato de práticas se não estiverem articulados com uma ação mais ampliada e constante.

É preciso progredir nas ações de prevenção ao uso de álcool e outras drogas na América Latina como um todo. Tais ações precisam ser planejadas para médio e longo prazo e não podem ser realizadas para obter resultados imediatos, apesar da demanda da gestão, profissionais e população por respostas instantâneas. Por isso, a importância de pesquisas-intervenções que levem em conta metodologias de implementação ampliadas, participativas, levadas a cabo em rede e contextualizadas, com destaque para desafios e possibilidades de disseminação nos países latino-americanos.

Agradecimentos. À Secretaria de Saúde do Município participante por au- torizar a realização do estudo; aos acadêmicos de Psicologia da Universidade Federal de Juiz de Fora, que participaram do trabalho de campo e coleta dos dados; à Fundação de Amparo à Pesquisa do Estado de Minas Gerais (FAPEMIG) pelo financiamento do estudo através do Edital 09/2010, “Extensão em Interface com a Pesquisa 2010"; e ao Conselho Nacional de Desenvolvimento Científico e Tecnológico (CNPq) pela bolsa de produtividade em pesquisa.

Conflito de interesses. Nada declarado pelos autores.

\section{REFERÊNCIAS}

1. Organización Panamericana de la Salud. Epidemiología del uso de drogas en América Latina y el Caribe: un enfoque de salud pública. Washington: OPS; 2009.

2. Rehm J, Monteiro M. Alcohol consumption and burden of disease in the Americas: implications for alcohol policy. Rev Panam Salud Publica. 2005;18(4-5):241-8.

3. Kaner EFS, Dickinson HO, Beyer F, Pienaar E, Schlesinger C, Campbell F, et al. The effectiveness of brief alcohol interventions in primary care settings: a systematic review. Drug Alcohol Rev. 2009;28(3):301-23.

4. Williams EC, Johnson ML, Lapham GT, Caldeiro RM, Chew L, Fletcher GS, et al. Strategies to implement alcohol screening and brief intervention in primary care settings: a structured literature review. Psychol Addict Behav. 2011;25(2):206-14.

5. Swan A, Sciacchitano L, Berends L. Alcohol and other drug brief intervention in primary care. Fitzroy, Victoria: Turning Point Alcohol and Drug Centre; 2008.

6. Organización Panamericana de la Salud. Renovación de la atención primaria de salud en las Américas: documento de posición de la Organización Panamericana de la Salud/ Organización Mundial de la Salud (OPS/ OMS). Washington, D.C: OPS, 2007.

7. Brasil, Ministério da Saúde. Política Nacional de Atenção Básica. Brasília: Ministério da Saúde; 2006. Disponível em: http://portal. saude.gov.br/portal/arquivos/pdf/pactovo lume4.pdf Acessado em 9 de março de 2012.

8. World Health Organization ASSIST Working Group. The Alcohol, Smoking and Substance Involvement Screening Test (ASSIST): development, reliability and feasibility. Addiction. 2002;97(9):1183-94.

9. Babor TF, Mcree GB, Kassebaum MA, Grimaldi PL, Ahmed K, Bray J. Screening, Brief Intervention, and Referral to Treatment (SBIRT): toward a public health approach to the management of substance abuse. Subst Abus. 2007;28(3):7-30.
10. Moretti-Pires RO. Complexidade em Saúde da Família e formação do futuro profissional de saúde. Interface - Comunic Saude Educ. 2009;13(30):153-66.

11. Ceccim RB. Educação Permanente em Saúde: descentralização e disseminação de capacidade pedagógica na saúde. Cienc Saude Coletiva. 2005;10(4):975-86.

12. Ronzani TM, Ribeiro MS, Amaral MB, Formigoni MLO. Implantação de rotinas de rastreamento do uso de risco de álcool e de uma intervenção breve na atenção primária à saúde: dificuldades a serem superadas. Cad Saude Publica. 2005;21(3):852-61.

13. González MA, Saforcada E. El papel de la psicología en la evaluación del impacto de ciertas políticas públicas de salud. Em: Lellis M, ed. Psicología y políticas públicas de salud. Buenos Aires: Paidó, 2006. Pp. 183-225.

14. Meneses MPR. Desarrollo de recursos y redes sociales. Em: Saforcada E, Sarriera JC, eds. Enfoques conceptuales y en psicología comunitaria. Buenos Aires: Paidó, 2008. Pp. 75-90.

15. Albuquerque PC, Stotz EN. A educação popular na atenção básica à saúde no município: em busca da integralidade. Interface (Botucatu). 2004;8(15):259-74.

16. Saforcada E. El Concepto de Salud Comunitaria ¿Denomina solo un escenario de trabajo o también una nueva estrategia de acción en salud pública? Psicol Pesq. 2008;2(02):3-13.

17. Giesbrecht N, Ferris J. Community-based research initiatives in prevention. Addiction 1993;88(suppl 1):83-93.

18. DATASUS. Cadastro Nacional de Estabelecimentos de Saúde Disponível em: http://cnes. datasus.gov.br/. Acessado em 7 de março 2012.

19. DATASUS. Cadernos de Informações em Saúde. Disponível em: http://tabnet.data sus.gov.br/tabdata/cadernos/cadernosmap. htm Acessado em 7 de março 2012.

20. Arantes RC, Martins JLA, Lima MF, Rocha RMN, Silva RC, Villela WV. Processo saúdedoença e promoção da saúde: aspectos históricos e conceituais. Rev APS. 2008;11(2):189-98.
21. Minayo MCS. Trabalho de campo: contexto de observação, interação e descoberta. Em: Minayo MCS, ed. Pesquisa social: teoria, método e criatividade. Petrópolis: Vozes; 2011. Pp. 61-78.

22. Freitas MFQ. Inserção na comunidade e análise de necessidades: reflexões sobre a prática do psicólogo. Psicol Reflex Crit. 1998;11(1);175-89.

23. Montero M. Vidas paralelas: psicología comunitaria en Latinoamerica y en Estados Unidos. Em: Montero M, ed. Psicología social comunitária - teoria, método y experiencia. Guadalajara: Universidad de Guadalajara; 1994. Pp. 19-46.

24. Ronzani TM, Silva CM. O Programa Saúde da Família segundo profissionais de saúde, gestores e usuários. Cienc Saude Coletiva. 2008;13(1):23-34.

25. Infante A, Mata I, Lopez-Acuna D. Reforma de los sistemas de salud en América Latina y el Caribe: situación y tendencias. Rev Panam Salud Publica. 2000;8(1/2):13-20.

26. Crevelim MA, Peduzzi M. Participação da comunidade na equipe de saúde da família: é possível estabelecer um projeto comum entre trabalhadores e usuários?. Cienc Saude Coletiva. 2005;10(2):323-31.

27. Ronzani TM, Mota DCB, Souza ICW. Prevenção do uso de álcool na atenção primária em municípios do estado de Minas Gerais. Rev. Saude Publica. 2009;43(suppl 1):51-61.

28. Briceño-León R. El contexto político de la participación comunitaria en América Latina. Cad Saude Publica. 1998;14(suppl 2):141-7.

Manuscrito recebido em 9 de março de 2012. Aceito em versão revisada em 5 de dezembro de 2012. 
ABSTRACT Objective. To develop a methodology to implement practices of prevention against the use of alcohol and other drugs in the context of primary health care (PHC) that will contribute to the debate about policies and actions in Latin American countries.

\section{A methodology to implement preventive actions against harmful drug use in the context of primary health care in Latin America}

Methods. This intervention research project was carried out in a small/mediumsized Brazilian city. The development process was assessed through participant observation with the aim of adapting the methodology to local needs and identifying existing weaknesses and strengths with impact on implementation.

Results. A model was developed with six stages: initial contact and planning, diagnosis and mapping, sensitization, training, follow-up, and communication of results to participants. The following weaknesses were identified: limitation of resources (human, financial, infrastructural), limitations in the coverage and comprehensiveness of the assistance network, poor participation from physicians, training based on medicalized care, insufficient participation of health care management, insufficient involvement and participation of civil society, and few opportunities for participation of the population in the planning and execution of public policies. Strengths included the participation of community health agents and nurses in applying, organizing, and planning initiatives, in addition to the organization of educative and preventive actions in schools and communities by health care teams, suggesting that it is possible to implement screening, brief intervention, and referral to treatment (SBIRT) initiatives in the context of PHC in Latin America.

Conclusions. The methodology developed in this study can be useful for Latin American countries if local needs are taken into consideration. It should be noted, however, that results will only be observed in the mid- to long term, rather than strictly in the short term.

Key words Primary health care; community-based participatory research; substance abuse detection; Latin America. 